\title{
Exploration and Practice of the Inquiry-based Teaching Method of Fashion Draping Course
}

\author{
Chongrong Liu \\ Wuhan Textile University, Wuhan, 430070, China \\ email:amyliu66@163.com
}

Keywords: The Inquiry-based Teaching Method; Fashion Draping Course; Practice

\begin{abstract}
Starting from the necessity of the inquiry-based teaching of fashion draping course, this paper focuses on the elaboration of the basic practice process of inquiry-based teaching methods in fashion draping course, and makes deep discussion on each relevant step of inquiry-based teaching methods. Finally, it analyzes the fashion draping course in accordance with the practice effects of the inquiry-based teaching method.
\end{abstract}

\section{Introduction}

Fashion draping is a typical clothing specialized course combined technical theories and artistic practice. The Fashion draping teaching emphasizes on students' thinking of theoretical knowledge and the practical operation skills; while traditional "cramming" teaching method condenses or ignores the students' autonomous learning process. As a result, a kind of new model of teaching is in urgent need to arouse interests and cultivate exploration spirit. Based on the problems appeared in the teaching process of fashion draping, we brings the inquiry-based teaching methods into the practical teaching of the "fashion draping" course. Teachers has abandoned the direct and unidirectional way of teaching to transfer professional knowledge and emphasized on the "question oriented” teaching. Students learn knowledge and solve problems through "autonomous learning”, so as to reach a good teaching effect of knowledge internalization.

\section{Connotation of the inquiry teaching mode of the fashion draping course}

At present, domestic and foreign higher education have reached a consensus on the understanding of the "inquiry-based teaching methods"; namely the inquiry teaching is a form of teaching activity with" inquiry” as the basic characteristic. In inquiry-based teaching method, the teacher guides the students to discover problems autonomously and to develop thinking ability in the researching discussion to solve problems by themselves. In the teaching of fashion draping course, the teacher design the staged teaching activities in advance in accordance with the course syllabus and ask students to complete the fashion draping works practice in the form of "cooperation and team discussion”. Students reach the purpose of learning in inquiry practice through the practical operation and mutual exchange of the fashion draping program. Finally, though comparing with each other, the teacher makes analysis and explanation of students' works, so that students can realize the internalization and absorption of the knowledge points. 


\section{Design Practice process of the inquiry-based teaching of fashion draping course}

\section{a). Create the inquiry question situation}

The generation of questions is the starting point of stimulating students' interest. Therefore, the teacher should be good at creating the inquiry situation in teaching, encouraging students to ask questions, and cultivating students' initiative query.

For example, the teacher proposes such as question after fully interaction with students: why do you like this clothing works? What detailed characteristics of this draping work have brought to you? What design methods of stereoscopic model are used in this works? For the stereoscopic styles in this original works, where do you think need to be improved? Through the questions, it will inspire students' thoughts on the inquiry of fashion draping knowledge to carry out free discussion in teams.

\section{b). Establish the autonomous inquiry direction through teachers' guidance and inspiration}

Students can select the fashion draping target plan which they like and want to carry out autonomous inquiry practice. The teacher only guides students to discover the problems around the plan and seek for the solutions, rather than directly giving the ready-made knowledge. If conditions permit, the teacher can also encourage students to explore more specialized projects and guide them to expand the knowledge scope of the autonomous inquiry; for example, inquiry with "the clothing transformation" as the theme or "the flipped turn of fashion draping" as the theme, and so on. The change of the different inquiry contents can cultivate students' practical strain capacity in using the fashion draping skills.

\section{c). Team cooperation to push the interactive thinking}

Students make discussion in teams, select the fashion draping content scheme of team members, and determine the main tasks of each team member. With team as the unit, it can create learning opportunities of mutual observation, mutual discussion and mutual improvement for students. In this way, students will not only pay attention to their own clothing draping works, but will attach great importance on the stereoscopic draping process of other team members. The conscious participation in the discussion is the process of the mutual collision and integration of students' thoughts. Although this inquiry and discovery process is challenging, it is very important to students' study. In this process, the teacher should avoid to guide students to the existing conclusions, but only supplement relevant knowledge for them, inspire their ideas, introduce ways and clues, and guide students to compare and inquiry different fashion draping methods.

\section{d). Construct professional knowledge to achieve conclusion and improvement}

Students present their fashion draping works schemes in the class; then students in other teams and the teacher discuss the works: (1) What are the strengths of this fashion draping work? What are your most appreciate aspect of this works? (2) Where do you think in this works needs to be improved? The teacher points out the problems and solutions in comments, summarizes the characteristics of teach team, and guide students to learn from others' strengths to improve their own design, so as to further verify and consolidate the acquired knowledge, thus reaching the construction of the complete meaning of knowledge. The figure.1 showed the whole process of the inquiry teaching of fashion draping course 


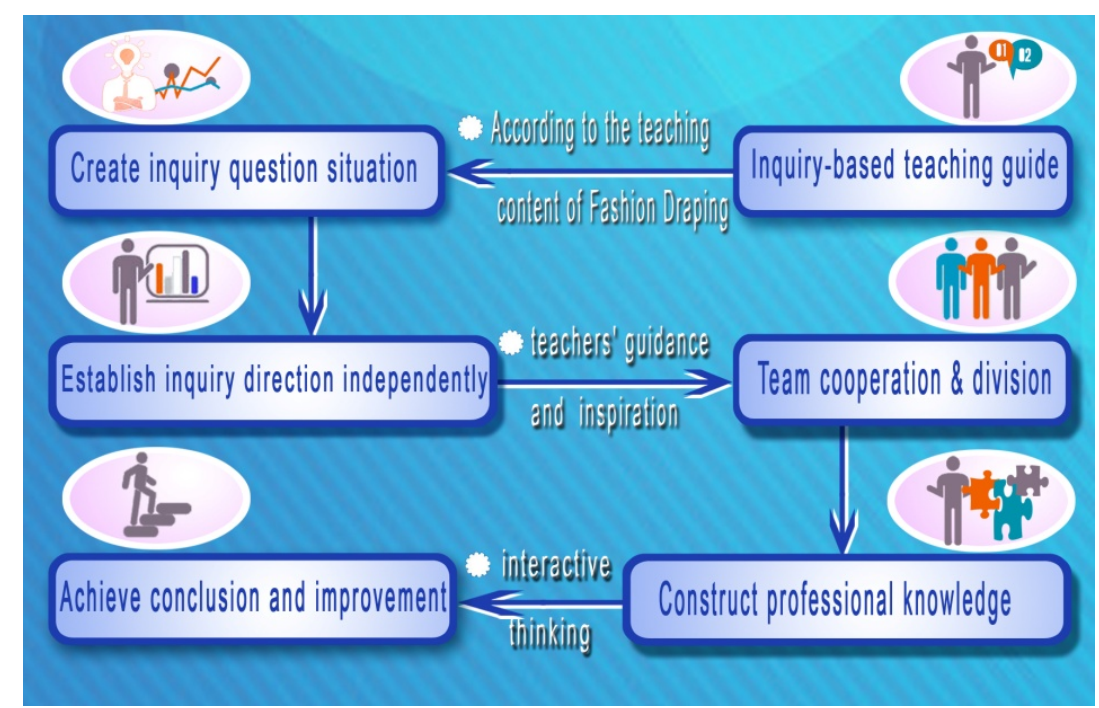

Figure.1. the process of the inquiry teaching of fashion draping course

\section{Test results}

In inquiry teaching, the teacher is the guider while the students are the inquirer. Based on the learning scheme, students discover the problems autonomously through individual inquiry, practical operation, team discussion, expression and communication. Through the self inquiry learning of seeking for the solutions, students conclude the modeling methods of fashion draping. As shown in figure.2, according to the statistical data of inquiry-based teaching methods, the inquiry-based teaching practice shows relatively good teaching effects in fashion draping course.

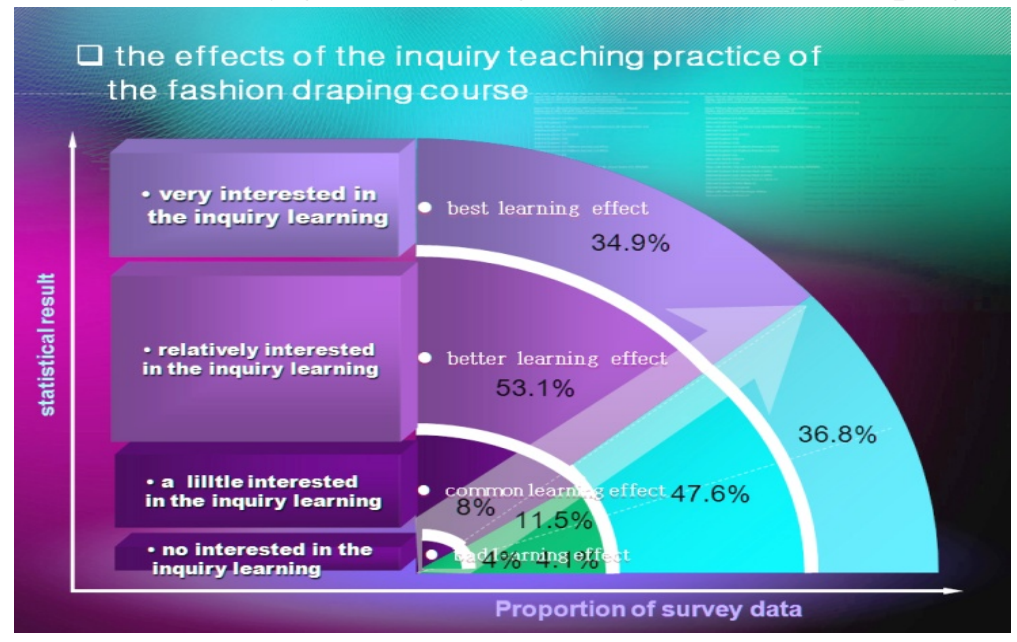

Figure.2. statistical table of the effect of the inquiry-based teaching practice

\section{Conclusion}

It can be concluded that the inquiry teaching practice of the fashion draping course can not only enable students to obtain the fashion draping professional knowledge, more importantly, it can cultivate students' inquiry and innovative ability, and enhance their independent learning ability. The teacher's guidance for students to establish the inquiry-based concept is also the cultivation process of students' adaptation ability required by their individual and social development, which is beneficial for students to improve their own innovative ability and analytical thinking ability. In this way, it extends and expands the classroom teaching, and achieves the cultivation goal to the maximum, namely cultivating students into the comprehensive professional talents. 


\section{Acknowledgements}

In this paper,This research was sponsored by the Teaching and research Foundation in Wuhan Textile University. (Project number: 2015 JY031; Project number: 2014TJKC028)

\section{References}

[1] Zainian Xue. Research and Practice on the Reform of Fashion draping Teaching Mode and Teaching Methods. Shandong Textile Economy, 2013.

[2] Shucong Chen. Exploration and Practice of Fashion draping Flipped Classroom Teaching Mode. Art and Design,2015.1

[3] Zuowei Zhang. Cultivation of Inforamtion Literacy Based on Inquiry-based Teaching, E-Business journal.2011.11:

[4] Wangqing Cai. Theory, Practice and Cases of Inquiry-based Teaching. Nanjing University Press.2015.5:178

[5] Zhongjin Huang. An Introduction to Education Policy. Beijing University Press.2011.11:87 